\title{
Timing of single balloon enteroscopy: significant or not?
}

Authors

Institutions
Kirbylee K. Nelson ${ }^{1}$, Seth Lipka², Ashley H. Davis-Yadley', Andrea C. Rodriguez ${ }^{1}$, Vignesh Doraiswamy ${ }^{3}$, Roshanak Rabbanifard ${ }^{2}$, Ambuj Kumar ${ }^{4}$, Patrick G. Brady ${ }^{2}$

Institutions are listed at the end of article. submitted

2. August 2015

accepted after revision

20. April 2016

\section{Bibliography}

DOI http://dx.doi.org/

10.1055/s-0042-108189

Published online: 29.6.2016

Endoscopy International Open

2016; 04: E761-E766

(c) Georg Thieme Verlag KG

Stuttgart $\cdot$ New York

E-ISSN 2196-9736

Corresponding author

Kirbylee K. Nelson, MD

Department of Internal

Medicine

University of South Florida

Morsani College of Medicine

12901 Bruce B. Downs Blvd

Tampa

FL 33612

USA

Fax: +1-813-259-0697

knelson1@health.usf.edu
Background: The development of balloon assisted enteroscopy (BAE) has revolutionized diagnostic and therapeutic modalities for small-bowel disorders. Although the role of emergent esophagogastroduodenoscopy and colonoscopy for upper and lower gastrointestinal bleeding is well defined, there is scarce data with regard to emergent BAE for gastrointestinal bleeding.

Study: We performed a retrospective cohort study including 110 hospitalized patients with obscure gastrointestinal bleeding who underwent single balloon enteroscopy (SBE) between January 2010 and August 2013. Patients were divided into two groups based on procedures performed emergently (within 24 hours) versus nonemergently (greater than 24 hours). Data on patient demographics, hemodynamic characteristics, type of obscure bleed, lesions identified, location of lesions, endoscopic intervention performed, need for further surgical or radiological intervention, diagnostic and therapeutic yield, and adverse events were compared between groups. Independent samples $t$ test and Fisher's exact test were used to assess the association between dependent and independent variables. For continuous data, the results were summarized as mean difference and 95\% confidence intervals

\section{Introduction}

\section{$\nabla$}

The small intestine has long been a challenge for gastroenterologists to examine using traditional endoscopic and radiologic techniques. The development of capsule endoscopy and balloon assisted enteroscopy (BAE) allows direct visualization of the entire small bowel with comparable diagnostic yields [1,2]. Whereas video capsule endoscopy (VCE) is limited to purely diagnostic ability, BAE allows histopathological diagnosis and treatment $[3,4]$. While VCE is generally the preferred initial diagnostic test because it is less invasive and more cost-effective, it is reasonable to per-
(CI), and for binary as odds ratio and $95 \% \mathrm{CI}$.

Results: Although patients in the group where enteroscopy was performed within 24 hours had a significantly higher incidence of radiological intervention ( $10.0 \%$ vs. $0.0 \%, P=0.019)$, the diagnostic and therapeutic yields between the two groups were not significantly different. Additionally, there were no statistically significant differences between the groups for overt and occult bleeding, transfusion requirements, type and location of lesions, endoscopic intervention performed, or adverse events. Hospital stay was shorter in the patients who had SBE within 24 hours of admission (6.2 vs. 11.3 days, $P<0.001$ ).

Conclusions: Although the diagnostic and therapeutic yields of SBE were not significantly different between patients having the procedure within 24 hours and those having it later, the early SBE group required more interventional radiology procedures. While endoscopists may not necessarily have to perform emergent assessment within 24 hours in patients with obscure gastrointestinal bleeding (OGIB) for greater diagnostic or therapeutic yield, early intervention may allow for earlier stabilization and thus shorter hospital stays. Prospective studies further evaluating these findings are indicated.

form BAE first if biopsies are needed or therapeutic intervention is probable [5-10].

The development of techniques allowing deep enteroscopy has drastically impacted our approach to the diagnosis and management of patients with obscure gastrointestinal bleeding (OGIB). OGIB is the most common indication for enteroscopy and is defined as occult or overt bleeding of unknown origin that persists or recurs after an initial negative evaluation with bidirectional endoscopy [11-14]. This technology has largely replaced conventional barium studies and intraoperative enteroscopy [15]. Although less data has been published with regard to single balloon 
enteroscopy (SBE) outcomes compared with double balloon enteroscopy (DBE), as it is a newer technology, multiple studies have shown the two devices to be comparable [12-14,16-21]. Our institution uses SBE for evaluation and therapeutic intervention of suspected small-bowel disorders. Currently, there is no consensus on the timing of enteroscopy in the evaluation of OGIB. The goal of this study was to determine whether there was an increased diagnostic or therapeutic yield of SBE performed emergently (within 24 hours of hospitalization) as opposed to non-emergently (more than 24 hours after admission) in patients with OGIB. To our knowledge, this study provides the largest cohort of patients undergoing SBE to evaluate OGIB in a single center.

\section{Materials and methods}

$\nabla$

\section{Definition}

In this study, we evaluated patients with overt bleeding and occult bleeding that developed with or without recurrence after initial negative esophagogastroduodenoscopy (EGD) and colonoscopy [11]. Overt bleeding was defined as visible gastrointestinal bleeding of unknown origin. Occult bleeding was gastrointestinal blood loss without visible bleeding defined by iron deficiency anemia or a positive fecal occult blood test. An emergent SBE was defined as SBE performed within 24 hours of hospitalization whereas non-emergent SBE was defined as SBE performed more than 24 hours after admission. The timing of endoscopy, emergent or non-emergent, was a clinical decision made by the endoscopist based on the patient's presentation and clinical status. VCE was not always performed immediately before enteroscopy, depending on the clinical setting. Capsules were not performed in the setting of a patient with an enteroscopy performed within 24 hours. This typically included patients with a high clinical suspicion for small-bowel pathology such as a history of known small-bowel arteriovenous malformations (AVMs), recent EGD or colonoscopy performed without yield, or positive nuclear medicine bleeding scans.

\section{Patients}

We obtained Institutional Review Board approval from the University of South Florida and Tampa General Hospital. Between January 2010 and August 2013, a total of 428 SBEs were performed on 377 patients with suspected small-bowel diseases. Of those patients, 110 consecutive hospitalized patients with obscure gastrointestinal bleeding were retrospectively reviewed. The other 267 patients underwent SBE as an outpatient or for non-bleeding indications and were excluded. Outpatients were not included, as this would lead to bias, because non-hospitalized patients typically do not carry the same morbidity as hospitalized patients. Basic demographic information was collected, which consisted of age, sex, and prior abdominal surgery. Length of hospital stay, hemodynamic characteristics, American Society of Anesthesiologists (ASA) physical classification system, type of obscure bleed, and need for emergent intervention were recorded. Hemodynamic characteristics included lowest reported hemoglobin, the need for transfusion, and units of blood transfused. Emergent intervention included procedures performed by surgery or interventional radiology. Endoscopic reports were examined for enteroscopy approach, location of lesion, type of lesion identified, therapeutic intervention performed, and adverse events.

\section{SBE procedure}

A SIF-Q180 enteroscope (Olympus Medical, Center Valley, PA, United States) was used to examine the small intestine according to the standard push-and-pull technique. SBE was performed by one of four experienced endoscopists with well over 100 SBEs per endoscopist. Training included either an advanced endoscopy fellowship and/or expertise using push enteroscopy before SBE. The initial approach to SBE was determined by clinical symptoms and findings on imaging or VCE. The oral approach was chosen first in patients with melena or localization of a lesion in the first $75 \%$ of the small bowel. The anal approach was chosen first in patients with hematochezia or evidence of a lesion in the distal $25 \%$. If clinical suspicion was high despite negative findings on the initial insertion route, a submucosal tattoo was placed to mark the deepest insertion point, and the other enteroscopic route was performed. Air insufflation was used in the majority of cases secondary to limited carbon dioxide availability. Overnight fasting was required of all patients. Additionally, those who underwent the retrograde approach received a bowel preparation with $4 \mathrm{~L}$ of polyethylene glycol electrolyte lavage solution the day before examination. Informed consent was obtained from each patient before the procedure. All procedures were performed under monitored anesthesia care using intravenous propofol administered by an anesthesiologist or nurse anesthetist.

\section{Assessment of endoscopic findings}

The location of the lesion was documented as occurring in the duodenum, jejunum, or ileum. We categorized the source of small-bowel bleeding as an erosion, ulcer, stricture, angiodysplasia/arteriovenous malformation (AVM), polyp, mass, Dieulafoy's lesion, varix, or other. The category "other" encompassed erythematous mucosa, a pigmented lesion, or an active bleeding site with no lesion identified. We did not consider lymphangiectasia to be a bleeding source.

\section{Endoscopic treatment}

Therapeutic intervention included argon plasma coagulation, multipolar electrocautery (Gold Probe), clipping, dilation, polypectomy, or dual therapy.

\section{Statistical analysis}

The association between dependent and independent variables was assessed using either an independent samples $t$ test for continuous variables or Fisher's exact test for binary variables. For continuous data, the results were summarized as mean difference (MD) and 95\% confidence intervals (CI). For dichotomous data, results were summarized using odds ratio (OR) and 95\%CI. Bonferroni correction was applied to adjust for multiple comparisons. $P$ values less than 0.05 were considered to be statistically significant. All data analyses were performed using SPSS v22 software.

\section{Results \\ $\boldsymbol{\nabla}$}

\section{Demographic and clinical characteristics}

Our study included 110 consecutive hospitalized patients with obscure gastrointestinal bleeding who underwent SBE. Patients were divided into two groups based on procedures performed emergently (within 24 hours) versus non-emergently (more than 24 hours). Enteroscopy was performed emergently in 30 patients $(27 \%)$ and non-emergently in 80 patients (73\%). VCE was 
Table 1 Demographic and hemodynamic characteristics of hospitalized patients with obscure gastrointestinal bleeding who underwent single balloon enteroscopy emergently (<24 hours) versus non-emergently ( 224 hours).

\begin{tabular}{|c|c|c|c|c|}
\hline & $<24$ hours $(n=30)$ & $\geq 24$ hours $(n=80)$ & OR $(95 \% \mathrm{Cl})$ or $\mathrm{MD}(95 \% \mathrm{Cl})$ & $P$ value \\
\hline Age, y & $63.1 \pm 13.4$ & $65.1 \pm 14.3$ & $-2.06(-7.9,3.8)$ & 0.48 \\
\hline Sex (female: male) & $14 / 30(46.7 \%)$ & $35 / 80(43.8 \%)$ & $1.13(0.49,2.61)$ & 0.78 \\
\hline History of abdominal surgery & $14 / 30(46.7 \%)$ & $34 / 80(42.5 \%)$ & $1.18(0.51,2.8)$ & 0.70 \\
\hline Days hospitalized & $6.2 \pm 5.7$ & $11.3 \pm 9.0$ & $-5.09(-8.0,-2.1)$ & 0.001 \\
\hline ASA status & $2.93 \pm 0.640$ & $3.00 \pm 0.585^{*}$ & $-0.067(-0.323,-0.190)$ & 0.61 \\
\hline Lowest hemoglobin reported, g/dL & $8.7 \pm 2.3$ & $8.7 \pm 2.2$ & $0.03(-1.05,1.11)$ & 0.96 \\
\hline Transfusions & $17 / 30(56.7 \%)$ & $41 / 80(51.2 \%)$ & $1.24(0.53,2.90)$ & 0.61 \\
\hline Emergent intervention (surgical or radiological) & $3 / 30(10.0 \%)$ & $0 / 80(0 \%)$ & $20.49(1.03,409.37)$ & 0.019 \\
\hline Units transfused & $1.93 \pm 2.31$ & $2.3 \pm 2.9$ & $-0.33(-1.42,0.75)$ & 0.55 \\
\hline
\end{tabular}

Values in italics are statistically significant $(P<0.05)$.

* In the $\geq 24$-hour group, ASA score was only able to be obtained in 77 of 80 patients.

Table 2 Diagnostic yield, therapeutic yield, enteroscopy approach, and adverse events of hospitalized patients with obscure gastrointestinal bleeding who underwent single balloon enteroscopy emergently (<24 hours) versus non-emergently ( $\geq 24$ hours). The diagnostic and therapeutic yields were calculated for all patients in each group as well as for overt and occult bleeding individually.

\begin{tabular}{|c|c|c|c|c|}
\hline & $<24$ hours $(n=30)$ & $\geq 24$ hours $(n=80)$ & OR (95\%Cl) or MD (95\%Cl) & $P$ value \\
\hline Diagnostic yield & $16 / 30(53.3 \%)$ & $50 / 80(62.5 \%)$ & $0.69(0.29,1.6)$ & 0.38 \\
\hline Therapeutic yield & $9 / 30(30 \%)$ & $34 / 80(42.5 \%)$ & $0.58(0.24,1.4)$ & 0.23 \\
\hline Overt & $21 / 30(70 \%)$ & $42 / 80(52.5 \%)$ & $2.11(0.86,5.2)$ & 0.10 \\
\hline Diagnostic yield & $11 / 21(52.4 \%)$ & $26 / 42(61.9 \%)$ & $0.68(0.24,1.95)$ & 0.47 \\
\hline Therapeutic yield & $6 / 21(28.6 \%)$ & $21 / 42(50 \%)$ & $0.40(0.13,1.23)$ & 0.11 \\
\hline Occult & $9 / 30(30 \%)$ & $38 / 80(47.5 \%)$ & $0.47(0.19,1.16)$ & 0.10 \\
\hline Diagnostic yield & $5 / 9(55.6 \%)$ & $24 / 38(63.2 \%)$ & $0.73(0.17,3.17)$ & 0.67 \\
\hline Therapeutic yield & $3 / 9(33.3 \%)$ & $13 / 38(34.2 \%)$ & $0.96(0.21,4.5)$ & 0.96 \\
\hline Anterograde approach & $29 / 30(96.7 \%)$ & $69 / 80(86.2 \%)$ & $4.62(0.57,37.5)$ & 0.15 \\
\hline Retrograde approach & $2 / 30(6.7 \%)$ & $13 / 80(16.2 \%)$ & $0.37(0.08,1.74)$ & 0.21 \\
\hline Adverse events & $2 / 30(6.7 \%)$ & $1 / 80(1.2 \%)$ & $0.18(0.02,2.03)$ & 0.16 \\
\hline
\end{tabular}

not performed before enteroscopy in the emergent group. In the non-emergent group, 57.5\% (46/80) underwent VCE initially. Patient demographics and hemodynamic characteristics are shown in Table 1. Mean age, gender, history of abdominal surgery, and ASA class were similar between the two groups. The majority of our patients in both groups were classified as ASA class III. Hospital stay was statistically significantly shorter in the patients who had SBE within 24 hours of admission versus after 24 hours $(6.2$ vs. 11.3 days, respectively; $P<0.001$ ). There was no statistically significant difference between groups with regard to lowest reported hemoglobin, the need for transfusion, and units of blood transfused.

Patients in the group where enteroscopy was performed within 24 hours versus greater than 24 hours had a significantly higher incidence of radiological intervention ( $10.0 \%$ vs. $0.0 \%$, respectively; $P=0.019$ ). Interventions included placement of an inferior vena cava (IVC) filter in a patient with a newly discovered clot and active gastrointestinal bleeding, pullback cholangiogram and replacement of an internal/external biliary drainage catheter in a patient with hemobilia induced by the initial drain, and placement of a gastrostomy tube for gastric decompression in a patient with a duodenal mass. The patient requiring an IVC filter was bleeding secondary to an AVM. The patient with hemobilia was presumed to be bleeding from a hepatic artery injury caused by placement of the initial drain. The patient with a duodenal mass requiring gastrostomy tube placement had no identified source of bleed.

\section{Characteristics of SBE}

Characteristics of SBE are displayed in Table 2. We examined the small intestine via the oral approach in 29 patients in the emergent group and 69 patients in the non-emergent group and via the anal approach in two patients in the emergent group and 13 patients in the non-emergent group. This included one patient in the emergent group and two patients in the non-emergent group who were examined via both oral and anal approaches (total enteroscopy). There was no significant difference in antegrade or retrograde approach between groups. When obscure bleeds were further broken up into overt and occult bleeding, there was no statistically significant difference with regard to timing of enteroscopy.

\section{Diagnostic yield}

The diagnostic yield was determined in the emergent and nonemergent groups for all bleeds, and for overt and occult bleeds individually ( Table 2 ). Among the 30 patients who underwent emergent enteroscopy, 16 were identified as having potential sources of small intestinal bleeding, for an overall diagnostic yield of $53.3 \%$. When further broken down by type of obscure bleed, the diagnostic yield was $52.4 \%(11 / 21)$ for overt bleeds and $55.6 \%$ (5/9) for occult bleeds. For the 80 patients in the nonemergent group, 50 had a potential bleeding source, for a diagnostic yield of $62.5 \%$. Of those 50 patients, the diagnostic yield was $61.9 \%$ (26/42) for overt bleeds, and 63.2\% (24/38) for occult bleeds. There was no statistically significant difference in diagnostic yield when comparing emergent and non-emergent SBE for all OGIB or overt versus occult individually. 


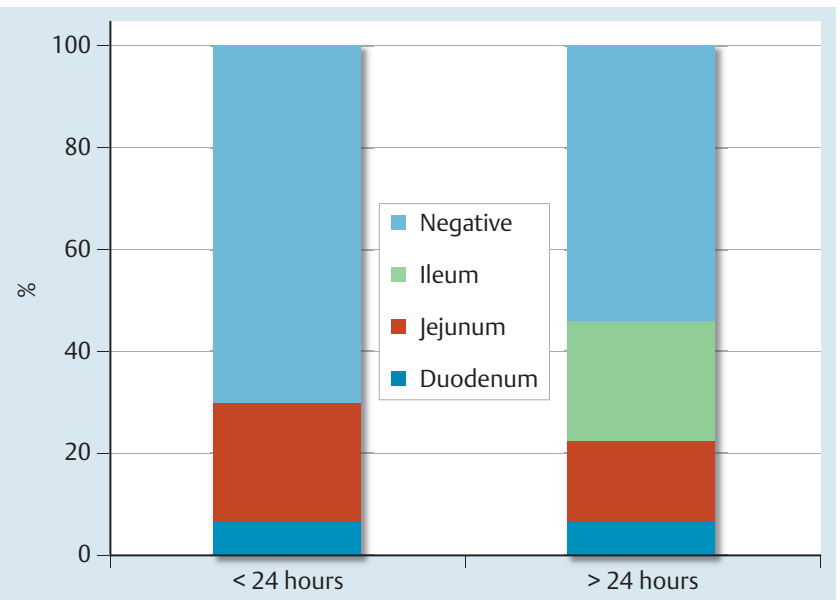

Fig. 1 Location of small-bowel lesions in hospitalized patients with obscure gastrointestinal bleeding who underwent single balloon enteroscopy emergently (<24 hours) versus non-emergently ( $>24$ hours).

\section{Therapeutic yield}

Similarly, the overall therapeutic yield and the therapeutic yield for overt and occult bleeding were calculated in the emergent and non-emergent groups ( $\boldsymbol{Q}$ Table 2 ). In the emergent group, 9/30 patients had therapeutic intervention, for an overall therapeutic yield of $30 \%$, with a therapeutic yield of $28.6 \%$ (6/21) for overt bleeds and $33.3 \%$ (3/9) for occult bleeds. Non-emergently, $34 / 80$ patients underwent therapeutic maneuvers, for an overall therapeutic yield of $42.5 \%$, with a therapeutic yield of $50 \%$ (21/ 42 ) for overt bleeds and $34.2 \%$ (13/38) for occult bleeds. The therapeutic yield between the two groups was not significantly different for all OGIB or by type of obscure bleeding specifically.

\section{Enteroscopy findings and therapeutic intervention}

Location of isolated lesions was similar between the emergent and non-emergent groups, as summarized in $\bullet$ Fig. 1. In both groups, lesions were most commonly found in the jejunum with the fewest lesions in the ileum. The potential bleeding sources are displayed in $\bullet$ Table 3 . When findings were present, bleeds were most likely due to ulcer $(23.3 \%$ ) when performed emergently and angiodysplasia/AVM (30.0\%) when performed non-emergently. Lesions outside the small bowel as possible sources of gastrointestinal bleeding were found in 21 of the 110 patients (19.1\%).
This included three patients with two different types of lesions, totaling 24 lesions detected. Findings included ulcer $(n=4)$, Cameron's lesions $(n=2)$, gastric antral vascular ectasias $(n=3)$, erosive gastritis $(n=8)$, and angiodysplasia/AVM $(n=7)$. Therapeutic interventions are shown in $\odot$ Fig. 2. Argon plasma coagulation and multipolar electrocautery were the primary modalities in both groups.

\section{Adverse events}

Adverse events were seen in three patients with no significant difference in the emergent and non-emergent groups, as seen in - Table 2. In the emergent group, two patients had adverse events secondary to transient hypoxia in one patient and shortness of breath, hypertension, and hyperglycemia warranting intensive care unit admission in the other patient. In the nonemergent group, one patient had mucosal trauma secondary to the overtube.

\section{Discussion \\ $\nabla$}

Review of the literature yields limited data with regard to the role of emergent SBE in the evaluation of OGIB [22,23]. Overall, there is limited data with regard to either emergent DBE or SBE to evaluate any symptomology. A small retrospective analysis of $10 \mathrm{pa}-$ tients by Mönkemüller et al. showed emergency DBE to be feasible and beneficial in facilitating diagnosis and therapy in OGIB [24]. Aniwan et al. looked specifically at patients with overt bleeding and found higher diagnostic and therapeutic yields when DBE was performed within 72 hours [25]. There are no similar studies evaluating the timing of DBE for both overt and occult gastrointestinal bleeding.

Regarding SBE, one recent study evaluated the impact of emergency SBE on the diagnosis and treatment of active overt OGIB. Pinto-Pais et al. retrospectively reviewed 43 patients with overt OGIB and subsequently divided them into active-overt and inactive-overt bleeders who underwent emergency and elective SBE, respectively [26]. Emergency SBE was defined as endoscopy performed within 24 hours of clinical presentation. Patients with occult OGIB were excluded. They found a significantly higher diagnostic yield in the emergency SBE group compared to the elective SBE group, $83.3 \%$ vs. $64.3 \%(P=0.038)$.

Currently, there is no consensus on the timing of enteroscopy in the evaluation of OGIB. While emergent has yet to be defined,

Table 3 Type of lesion identified in hospitalized patients with obscure gastrointestinal bleeding who underwent single balloon enteroscopy emergently $(<24$ hours) versus non-emergently ( 224 hours).

\begin{tabular}{|c|c|c|c|c|}
\hline & $<24$ hours $(n=30)$ & $\geq 24$ hours $(n=80)$ & OR $(95 \% \mathrm{Cl})$ or $\mathrm{MD}(95 \% \mathrm{Cl})$ & $P$ value \\
\hline Erosion & $1 / 30(3.3 \%)$ & $5 / 80(6.2 \%)$ & $0.52(0.06,4.62)$ & 0.56 \\
\hline Ulcer & $7 / 30(23.3 \%)$ & $14 / 80(17.5 \%)$ & $1.44(0.52,4.00)$ & 0.49 \\
\hline Stricture & $1 / 30(3.3 \%)$ & $4 / 80(5.0 \%)$ & $0.66(0.07,6.10)$ & 0.71 \\
\hline Angiodysplasia/AVM & $5 / 30(16.7 \%)$ & $24 / 80(30.0 \%)$ & $0.47(0.16,1.36)$ & 0.16 \\
\hline Polyp & $2 / 30(6.7 \%)$ & $8 / 80(10 \%)$ & $0.64(0.13,3.22)$ & 0.59 \\
\hline Mass & $0 / 30$ & $1 / 80(1.2 \%)$ & N/A & N/A \\
\hline Dieulafoy's lesion & $2 / 30(6.7 \%)$ & $0 / 80$ & N/A & N/A \\
\hline Varix & $0 / 30$ & $2 / 80(2.5 \%)$ & $\mathrm{N} / \mathrm{A}$ & $\mathrm{N} / \mathrm{A}$ \\
\hline Other & $2 / 30(6.7 \%)$ & $13 / 80(16.2 \%)$ & $0.37(0.08,1.74)$ & 0.21 \\
\hline Negative & $11 / 30(36.7 \%)$ & $26 / 80(32.5 \%)$ & $1.20(0.50,2.89)$ & 0.68 \\
\hline
\end{tabular}

AVM, arteriovenous malformation. 

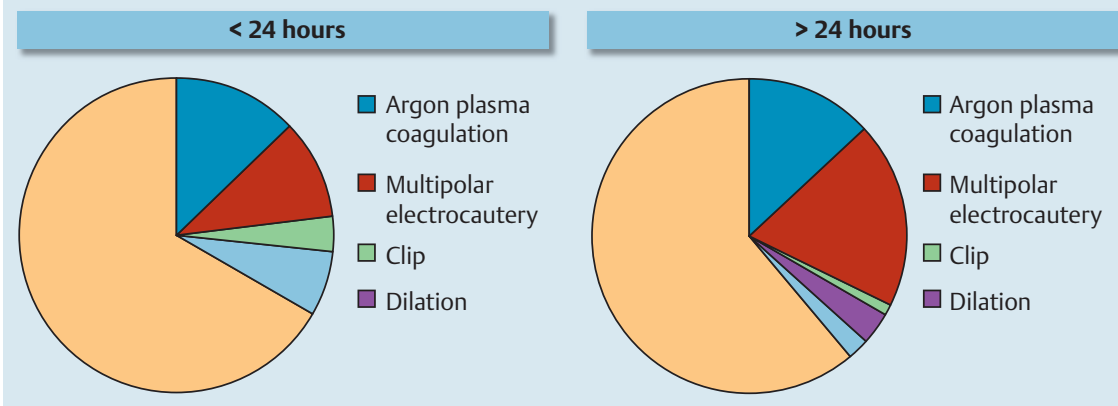

Fig. 2 Therapeutic interventions performed in hospitalized patients with obscure gastrointestinal bleeding who underwent single balloon enteroscopy emergently ( $<24$ hours) versus non-emergently (>24 hours).
Mönkemüller et al. and Pinto-Pais et al. suggested that the term applies to any procedure performed within 24 hours of clinical presentation [24,26]. We, therefore, used 24 hours to define emergent endoscopy in our study.

Our study evaluated the impact of timing of SBE on the diagnostic yield, therapeutic yield, need for surgical or radiological intervention, and adverse events for both antegrade and retrograde enteroscopies. This data illustrates that patients in the group where enteroscopy was performed within 24 hours had a significantly higher incidence of radiological intervention $(10.0 \% \mathrm{vs}$. $0.0 \%$ ). The diagnostic and therapeutic yields between the two groups were not significantly different. Interestingly, though not statistically significant, the diagnostic and therapeutic yields were actually lower in the emergent group, $53 \%$ vs. $63 \%$ and $30 \%$ vs. $43 \%$, respectively. Our diagnostic yields are comparable to other published studies $(47-60 \%)[12,13,27,28]$. Variation in results was likely due to differences in inclusion criteria. Some studies included non-bleeding indications and/or both inpatients and outpatients $[12,27,28]$. Furthermore, only one study analyzed therapeutic yield in patients who underwent SBE to evaluate OGIB specifically [13]. Ramchandani et al. performed therapeutic intervention in $40 \%(16 / 40)$ of patients, with our study showing therapeutic yields of $30 \%$ and $43 \%$ in the emergent and non-emergent groups, respectively.

Of the patients requiring radiological intervention, AVM was the cause of bleeding in one patient with no lesion identified in the other two patients. Interventions included IVC filter placement, replacement of a biliary drainage catheter, and gastrostomy tube placement. The radiological interventions necessary were incidental and unrelated to whether or not enteroscopy was performed. There was no significant difference in adverse events with regard to timing of enteroscopy.

The secondary aim of this study was to compare patient demographics, ASA status, hemodynamic characteristics, type of obscure bleed, lesions identified, location of lesions, and endoscopic intervention performed between groups. Patient demographics (mean age, gender, and history of abdominal surgery), ASA status, hemodynamic characteristics (lowest reported hemoglobin, the need for transfusion, and units of blood transfused), and type of obscure bleed (overt vs. occult) were similar between the two groups. In both groups, lesions were most commonly found in the jejunum. When findings were present, bleeds were most likely due to ulcer (23.3\%) when performed emergently and angiodysplasia/AVM (30.0\%) when performed non-emergently, similar to other published studies on both DBE and SBE $[12,13$, $27-29$ ]. Only one mass, pathologically consistent with lipoma, was detected. We did not find any gastrointestinal stromal tumors (GISTs) or neuroendocrine tumors (NETs), likely because GISTs and NETs typically cause other symptoms aside from bleeding. Argon plasma coagulation and multipolar electrocautery were the primary modalities in both groups. Hospital stay was significantly shorter in the patients who had SBE within 24 hours of admission versus after 24 hours (6.2 vs. 11.3 days). This may be due to earlier diagnosis with earlier application of endoscopic and/or medical therapy, and earlier stabilization of hemoglobin levels.

Our study was limited due to its single center retrospective nature and small population size. There were a lower number of patients in the urgent group than in the non-urgent SBE group. This could be related to the variable reasons for hospital admission, with only a portion of the cohort initially presenting with OGIB. The majority of cases were anterograde based on clinical findings or imaging. Our study did not include long-term follow-up, therefore we cannot assess the extended impact of intervention on rebleeding rates with SBE. The strengths of our study include the evaluation of SBE in regards to timing of overt and occult bleeding, something not reported previously. This study was also performed by experienced gastroenterologists, with over 100 SBEs performed before the study.

\section{Conclusion}

This is the first study to evaluate the impact of timing of SBE, emergent (within 24 hours) versus non-emergent (greater than 24 hours), in hospitalized patients with OGIB. One prior study retrospectively evaluated active overt bleeds only [26]. This is the first study to include occult bleeders. It is also the largest cohort of patients who underwent SBE to evaluate OGIB in a single center reported to date. Currently, there is no consensus on the optimal timing of SBE in patients with obscure bleeding. Although patients in the group where enteroscopy was performed within 24 hours had a significantly higher incidence of radiological intervention, these occurrences were unrelated to whether or not enteroscopy was performed. The diagnostic and therapeutic yields between the two groups were not significantly different, however, the emergent SBE group had a significantly shorter hospital stay, which may be related to earlier diagnosis and therapy. This suggests that, while endoscopists may not necessarily need to perform emergent assessment within 24 hours in patients with OGIB for greater diagnostic or therapeutic yield, early intervention does allow for earlier stabilization of hemodynamics and thus shorter hospital stays. Prospective studies further evaluating these findings are indicated.

\section{Competing interests: None}


Institutions

${ }^{1}$ Department of Internal Medicine, University of South Florida Morsani College of Medicine, Tampa, FL, USA

2 Department of Internal Medicine, Division of Digestive Diseases and Nutrition, University of South Florida Morsani College of Medicine, Tampa, FL, USA

${ }^{3}$ University of South Florida Morsani College of Medicine, Tampa, FL, USA

${ }^{4}$ Department of Evidence Based Medicine and Outcomes Research, University of South Florida Morsani College of Medicine, Tampa, FL, USA

\section{References}

1 Elena RM, Riccardo U, Rossella $C$ et al. Current status of device-assisted enteroscopy: Technical matters, indication, limits and complications. World J Gastrointest Endosc 2012; 4: 453-461

2 Aktas H, Mensink PB. Small bowel diagnostics: current place of small bowel endoscopy. Best Pract Res Clin Gastroenterol 2012; 26: 209220

3 Nakamura M, Niwa Y, Ohmiya $N$ et al. Preliminary comparison of capsule endoscopy and double-balloon enteroscopy in patients with suspected small-bowel bleeding. Endoscopy 2006; 38: 59-66

4 Mazzarolo S, Brady P. Small bowel capsule endoscopy: a systematic review. South Med J 2007; 100: 274-280

5 Albert JG, Nachtigall F, Wiedbrauck $F$ et al. Minimizing procedural cost in diagnosing small bowel bleeding: comparison of a strategy based on initial capsule endoscopy versus initial double-balloon enteroscopy. Eur J Gastroenterol Hepatol 2010; 22: 679-688

6 Pohl J, Blancas JM, Cave D et al. Consensus report of the 2nd International Conference on double balloon endoscopy. Endoscopy 2008; 40: $156-160$

7 Teshima CW, May G. Small bowel enteroscopy. Can J Gastroenterol 2012; 26: 269-275

8 Albert JG, Schulbe R, Hahn L et al. Impact of capsule endoscopy on outcome in mid-intestinal bleeding: a multicentre cohort study in 285 patients. Eur J Gastroenterol Hepatol 2008; 20: 971-977

9 Teshima CW, Kuipers EJ, van Zanten SV et al. Double balloon enteroscopy and capsule endoscopy for obscure gastrointestinal bleeding: an updated meta-analysis. J Gastroenterol Hepatol 2011; 26: 796-801

10 Fisher L, Lee Krinsky M, Anderson MA et al. The role of endoscopy in the management of obscure GI bleeding. Gastrointest Endosc 2010; 72: $471-479$

11 Raju GS, Gerson L, Das A et al. American Gastroenterological Association (AGA) Institute medical position statement on obscure gastrointestinal bleeding. Gastroenterology 2007; 133: 1694-1696

12 Upchurch BR, Sanaka MR, Lopez AR et al. The clinical utility of singleballoon enteroscopy: a single-center experience of 172 procedures. Gastrointest Endosc 2010; 71: 1218 - 1223

13 Ramchandani M, Reddy DN, Gupta R et al. Diagnostic yield and therapeutic impact of single-balloon enteroscopy: series of 106 cases. J Gastroenterol Hepatol 2009; 24: 1631-1638
14 Efthymiou M, Desmond PV, Brown G et al. SINGLE-01: a randomized, controlled trial comparing the efficacy and depth of insertion of single- and double-balloon enteroscopy by using a novel method to determine insertion depth. Gastrointest Endosc 2012; 76: 972 -980

15 Pennazio $M$. Enteroscopy in the diagnosis and management of obscure gastrointestinal bleeding. Gastrointest Endosc Clin N Am 2009; 19: 409-426

16 Tsujikawa T, Saitoh Y, Andoh A et al. Novel single-balloon enteroscopy for diagnosis and treatment of the small intestine: preliminary experiences. Endoscopy 2008; 40: 11-15

17 Takano N, Yamada A, Watabe $H$ et al. Single-balloon versus double-balloon endoscopy for achieving total enteroscopy: a randomized, controlled trial. Gastrointest Endosc 2011; 73: 734-739

18 Domagk D, Mensink P, Aktas H et al. Single- vs. double-balloon enteroscopy in small-bowel diagnostics: a randomized multicenter trial. Endoscopy 2011; 43: $472-476$

19 Gerson LB. Long-term outcomes after single-balloon enteroscopy: are they any different from double-balloon enteroscopy for vascular lesions? Dig Dis Sci 2013; 58: 2441 - 2443

20 Kushnir VM, Tang M, Goodwin J et al. Long-term outcomes after singleballoon enteroscopy in patients with obscure gastrointestinal bleeding. Dig Dis Sci 2013; 58: $2572-2579$

21 Lipka S, Rabbanifard R, Kumar A et al. Single versus double balloon enteroscopy for small bowel diagnostics: a systematic review and metaanalysis. J Clin Gastroenterol 2015; 49: 177 -184

22 Cooper GS, Kou TD, Wong RC. Use and impact of early endoscopy in elderly patients with peptic ulcer hemorrhage: a population-based analysis. Gastrointest Endosc 2009; 70: 229-235

23 Niikura R, Nagata N, Aoki T et al. Predictors for identification of stigmata of recent hemorrhage on colonic diverticula in lower gastrointestinal bleeding. J Clin Gastroenterol 2015; 49: e24-e30

24 Mönkemüller K, Neumann H, Meyer F et al. A retrospective analysis of emergency double-balloon enteroscopy for small-bowel bleeding. Endoscopy 2009; 41: 715-717

25 Aniwan S, Viriyautsahakul V, Rerknimitr R et al. Urgent double balloon endoscopy provides higher yields than non-urgent double balloon endoscopy in overt obscure gastrointestinal bleeding. Endosc Int Open 2014; 2: E90-E95

26 Pinto-Pais T, Pinho R, Rodrigues A et al. Emergency single-balloon enteroscopy in overt obscure gastrointestinal bleeding: Efficacy and safety. United European Gastroenterol J 2014; 2: 490 - 496

27 Khashab MA, Lennon AM, Dunbar KB et al. A comparative evaluation of single-balloon enteroscopy and spiral enteroscopy for patients with mid-gut disorders. Gastrointest Endosc 2010; 72: 766 - 772

28 Frantz DJ, Dellon ES, Grimm IS et al. Single-balloon enteroscopy: results from an initial experience at a U.S. tertiary-care center. Gastrointest Endosc 2010; 72: 422-426

29 Dray X, Camus M, Coelho J et al. Treatment of gastrointestinal angiodysplasia and unmet needs. Dig Liver Dis 2011; 43: 515-522 